\title{
Segregation by color/luminance does not necessarily facilitate motion discrimination in the presence of motion distractors
}

\author{
HYUNG-CHUL O. LI \\ Kwangwoon University, Seoul, Korea \\ and \\ FREDERICK A. A. KINGDOM \\ McGill University, Montreal, Quebec, Canada
}

\begin{abstract}
Under what circumstances is the common motion of a group of elements more easily perceived when the elements differ in color and/or luminance polarity from their surround? Croner and Albright (1997), using a conventional global motion paradigm, first showed that motion coherence thresholds fell when target and distractor elements were made different in color. However, in their paradigm, there was a cue in the static view of the stimulus as to which elements belonged to the target. Arguably, in order to determine whether the visual system automatically groups, or prefilters, the image into different color maps for motion processing, such static form cues should be eliminated. Using various arrangements of the global motion stimulus in which we eliminated all static form cues, we found that global motion thresholds were no better when target and distractors differed in color than when they were identical, except under certain circumstances in which subjects had prior knowledge of the specific target color. We conclude that, in the absence of either static form cues or the possibility of selective attention to the target color, features with similar colors/luminance-polarities are not automatically grouped for global motion analysis.
\end{abstract}

An important function of vision is figure-ground segregation, which exploits the fact that objects typically differ from their surrounds along one or more dimensionfor example, color, luminance, orientation, scale, binocular disparity, and motion. Although, it is clear that any of these dimensions alone can support segregation, we currently know little about how they interact when more than one dimension differentiates figure from ground. For example, under what circumstances is movement perception improved when figure and ground differ in color? This study determines the circumstances in which a color difference between coherently moving target elements and incoherently moving distractors facilitates discrimination of the target's direction of motion.

The role of color vision in motion processing has primarily been examined at isoluminance - that is, with stimuli defined purely by color contrast. In general, motion perception is weaker at isoluminance than with luminance contrast present, but there is nevertheless good evidence for a chromatic input to at least some types of motion processing (Cavanagh \& Favreau, 1985; Cropper \& Derrington, 1996; Green, 1989; Morgan \& Ingle, 1994;

This research was supported by Medical Research Council of Canada Grant MT 11554, given to F.A.A.K. Correspondence concerning this article should be addressed to H.-C. O. Li, Department of Industrial Psychology, Kwangwoon University, Nowon-Gu, Wolgae-Dong, 447-1, Seoul, Korea (e-mail: hyung@ daisy.kwangwoon.ac.kr).
Mullen \& Baker, 1985; Palmer, Mobley, \& Teller, 1993; Papathomas, Gorea, \& Julesz, 1991; Saito, Tanaka, Isono, Yasuda, \& Mikami, 1989; Yoshizawa, Mullen, \& Baker, 2000). That color might facilitate motion processing by helping segregate target elements from distractors is, however, not necessarily contingent on motion perception being possible at isoluminance. Chromatic differences might be beneficial in conjunction with luminance contrast. Our primary interest is whether the addition of a color difference to a target already defined by luminance contrast facilitates the perception of its motion.

Recent studies by Edwards and Badcock $(1994,1996)$ and Croner and Albright (1997) have provided evidence relevant to this issue. These investigators employed the globalmotion paradigm, in which subjects were required to discriminate the direction of motion of a set of coherently moving target dots among incoherently moving distractor dots. As in nearly all studies of global motion, their stimuli were not isoluminant, even though the color of the luminance-contrast-defined dots was a key independent variable. The dependent variable was the proportion of target dots required for a criterion level of accuracy. In Edwards and Badcock's (1994) study, a comparison was made between thresholds obtained when all the dots were bright (i.e., both the target and the distractors) and those obtained when half the dots were bright and half dark, with the bright dots containing the target (see Figure 1). No significant differences between thresholds were found. Edwards and Badcock (1996) found a similar result when 
Different luminance

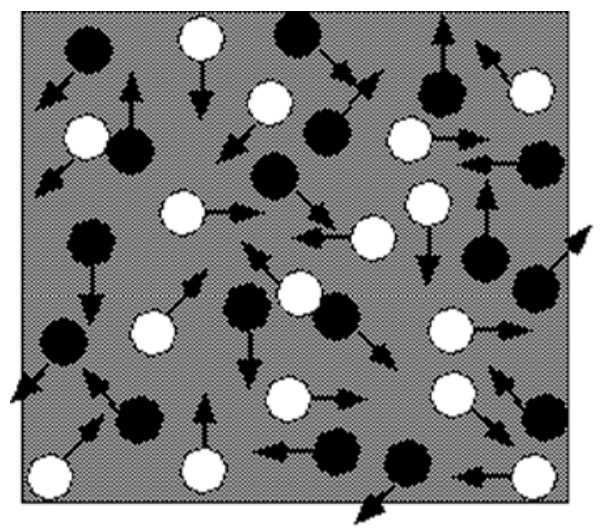

dark dot
Same luminance

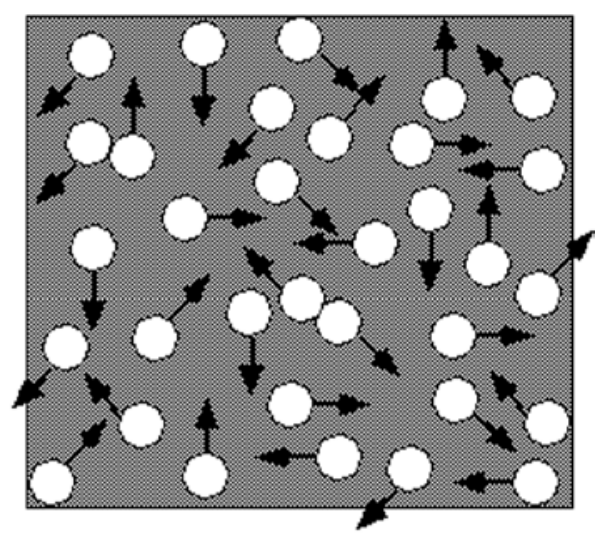

bright dot

Figure 1. Schematic diagram of the motion stimulus used in the Edwards and Badcock (1996) study. In the different-luminance condition, half the dots were bright and half dark, with the bright dots containing the target (which moves to the right). In the same-luminance condition, all the dots were bright (i.e., both the target and the distractors). In both conditions, coherent-motion direction discrimination was measured as a function of the proportion of target dots.

all the dots were red (with an $8 \%$ luminance contrast); Thresholds were no higher than when half were red and half green, the red dots containing the target. These two studies appeared to show that the visual system was unable to "ignore" the $50 \%$ of the dots that were pure distractors and different in color or luminance polarity from the target dots. It is important to understand, however, that in the Edward and Badcock mixed-dot conditions, target and distractor were only partially differentiated by dot type. For example, in the color experiment, the number of coherently moving dots at threshold was about $10 \%$. Therefore in the mixed-dot condition, although all the target dots were red, $44 \%$ of the distractor dots were red and $56 \%$ green. This rather small difference in the color composition of the distractor dots may have obscured any potential benefits of segregation.

Croner and Albright (1997) used stimuli constructed according to a different rule (see Figure 2). In their mixeddot conditions, the target and the distractor dots were completely segregated by color or luminance polarity. Thus, if thresholds were around $10 \%$ in the mixed-dot condition, the $10 \%$ of target dots were one color, and the $90 \%$ of distractor dots were another. Unlike Edwards and Badcock (1994, 1996), Croner and Albright (1997) found that performance in their mixed-dot conditions was significantly better than that in their uniform-dot conditions. They proposed that either preattentive segmentation or selective attention to the target dots underlay the beneficial effects of segregation. Edwards and Badcock (1996), discussing an earlier report by Croner and Albright (1994), also argued that selective attention underlay the Croner and Albright result. A recent study by Snowden and Edmunds (1999) that used a similar paradigm also supported the selective attention explanation. Here, we suggest that the critical stimulus characteristic in Croner and Albright's paradigm, which enabled selective attention to operate even without prior knowledge of the color of the target dots, was that the target dots were in a minority. As a result, they popped out from the background, even in a static view of the stimulus. We refer to this as a static form cue (see Figure 2). In the Edwards and Badcock stimuli, no such static form cue was present, because there were always equal numbers of the two colors in the mixed-dot condition, irrespective of the number of target dots (see Figure 1). A static view of one of the motion frames in Edwards and Badcock's mixed-dot condition would not unambiguously reveal the target dots.

The lack of an effect of segregation by color/luminance in the Edwards and Badcock $(1994,1996)$ studies suggests that the motion system does not automatically group, or prefilter, the image into separate color maps prior to motion analysis. However, there are other possible explanations. Croner and Albright (1997) suggested that Edwards and Badcock's (1994) negative finding with dots of different luminance polarities might be an artifact that was due to the different saliencies of the bright and the dark dots. They argued that the use of Weber contrast to equate the bright and the dark dots might have resulted in the dark dots being more salient. Thus in Edwards and Badcock's (1994) mixed-dot condition (half bright and half dark), in which the bright dots contained the target, the more salient, dark distractor dots may have undermined the potential advantage of having nearly half of the distractor dots different in polarity from the target. Another possible explanation for the lack of segregation in the Edwards and Badcock studies is the incomplete segregation of target and distractor by dot type that we alluded to earlier. 
Color-segregated: Popout

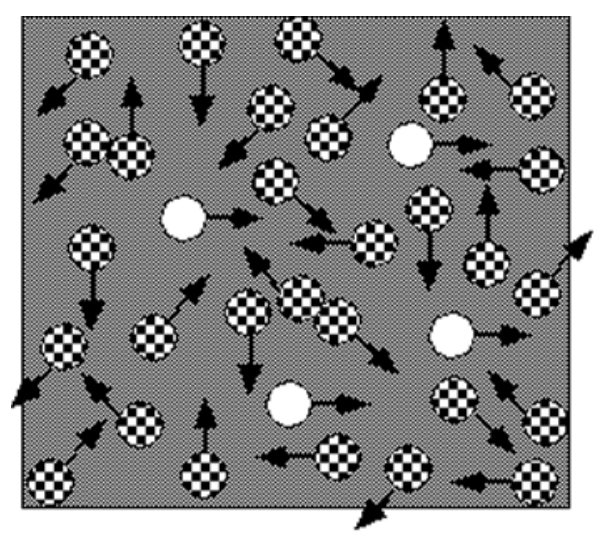

Color-uniform: Control

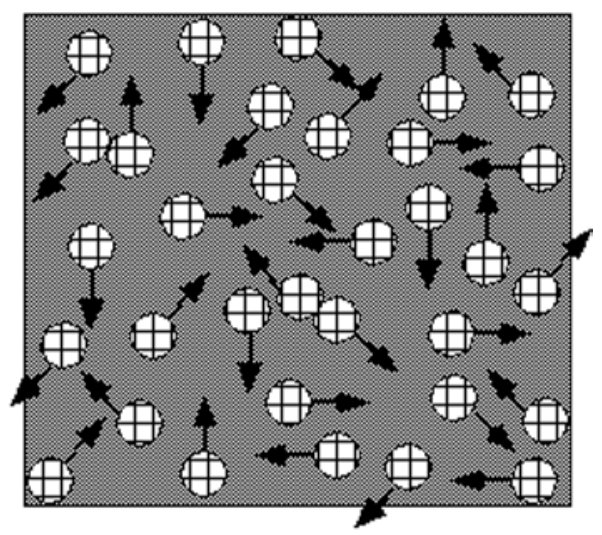

bright green

bright red

bright yellow

Figure 2. Schematic diagram of the motion stimuli used to replicate Croner and Albright (1997). In the color-segregated condition ("hetero-cue" in Croner \& Albright), all the target dots were of one color, either bright red or bright green, and all the distractor dots were the opposite color. In the color-uniform control condition ("homo-cue" in Croner \& Albright), all the dots were bright yellow. In both conditions, coherent-motion direction discrimination was measured as a function of the proportion of target dots.

We therefore decided to reexamine the role of color/ luminance as a segmentation cue in global motion. Specifically, we wanted to test the following hypotheses: (1) that the visual system does not automatically prefilter the image into separate color maps for motion analysis, and (2) that only when segregation introduces a static form cue to the target does motion perception benefit. To test these hypotheses, it was necessary to eliminate a number of alternative reasons for the differences in results between the Edwards and Badcock $(1994,1996)$ and the Croner and Albright $(1994,1997)$ studies.

The organization of the experiments is as follows. We first replicated the result of Croner and Albright (1997); this was necessary to establish whether their result (i.e., segregation by color/luminance facilitated motion discrimination) could be found with our stimuli. In all the subsequent experiments, we eliminated the static form cues that we argue constituted the hallmark of the Croner and Albright paradigm. The second experiment was similar to that employed by Edwards and Badcock (1994, 1996), in that there were equal numbers of the two dot types in the segregated condition. The important difference, however, is that we used an identical dot composition in both the test (segregated by dot type) and the comparison (nonsegregated by dot type) stimuli (see Figure 3). In addition, on every trial of the segregated condition, we randomized which dot type was target and which distractor. These manipulations minimized the effects of any difference in dot saliency and, in addition, prevented selective attention to the target dots. The third experiment tested whether the negative results of the Edwards and Badcock studies were simply a consequence of incomplete segregation of the target and the distractor by dot type. We set the proportion of target dots to 50\% (which produced complete segregation of target and distractor by dot type) and avoided a ceiling effect in performance by reducing the between-frame dot displacement size. Finally, in the fourth experiment, by fixing the target dot color throughout each session and encouraging the subjects to attend only to that color, we tested whether selective attention to the target dots could facilitate performance even when no static form cues were present.

The results of our experiments have served to refine our understanding of the conditions under which segregation by color/luminance facilitates the perception of global motion.

\section{GENERAL METHOD}

\section{Subjects}

The 2 authors, H.-C.L. and F.K., and 3 naive subjects, M.K., M.R., and E.O., participated. All had normal or corrected-tonormal vision. H.-C.L. and F.K. participated in all the experiments. M.K. and M.R. participated in Experiments 3 and 4. E.O. participated in Experiments 3B, 3C, and 4.

\section{Stimuli}

Generation and display. The stimuli were generated by a PowerMac 8500/180 with 8 bits-per-gun intensity resolution and were displayed on a 17-in. NEC MultiSync XV17+ RGB video monitor with P22 phosphors. Screen resolution was $640 \times 480$ pixels, and the frame rate was $120 \mathrm{~Hz}$ (noninterlaced). The screen nonlinearity was gamma corrected following calibration of the red and green gun luminances with a UDT photometer. C.I.E. coordinates were $x=$ $0.610, y=0.350$ for the red and $x=0.307, y=0.595$ for the green.

Gaussian dots. The Gaussian dots were generated using the following function:

$$
L(x, y)=M+A \exp \left[-\left(x^{2}+y^{2}\right) /\left(2 \sigma^{2}\right)\right],
$$

where $M$ was mean luminance (which depended on whether the red or the green gun was modulated), $A$ was the amplitude of $50 \%$ (i.e., 

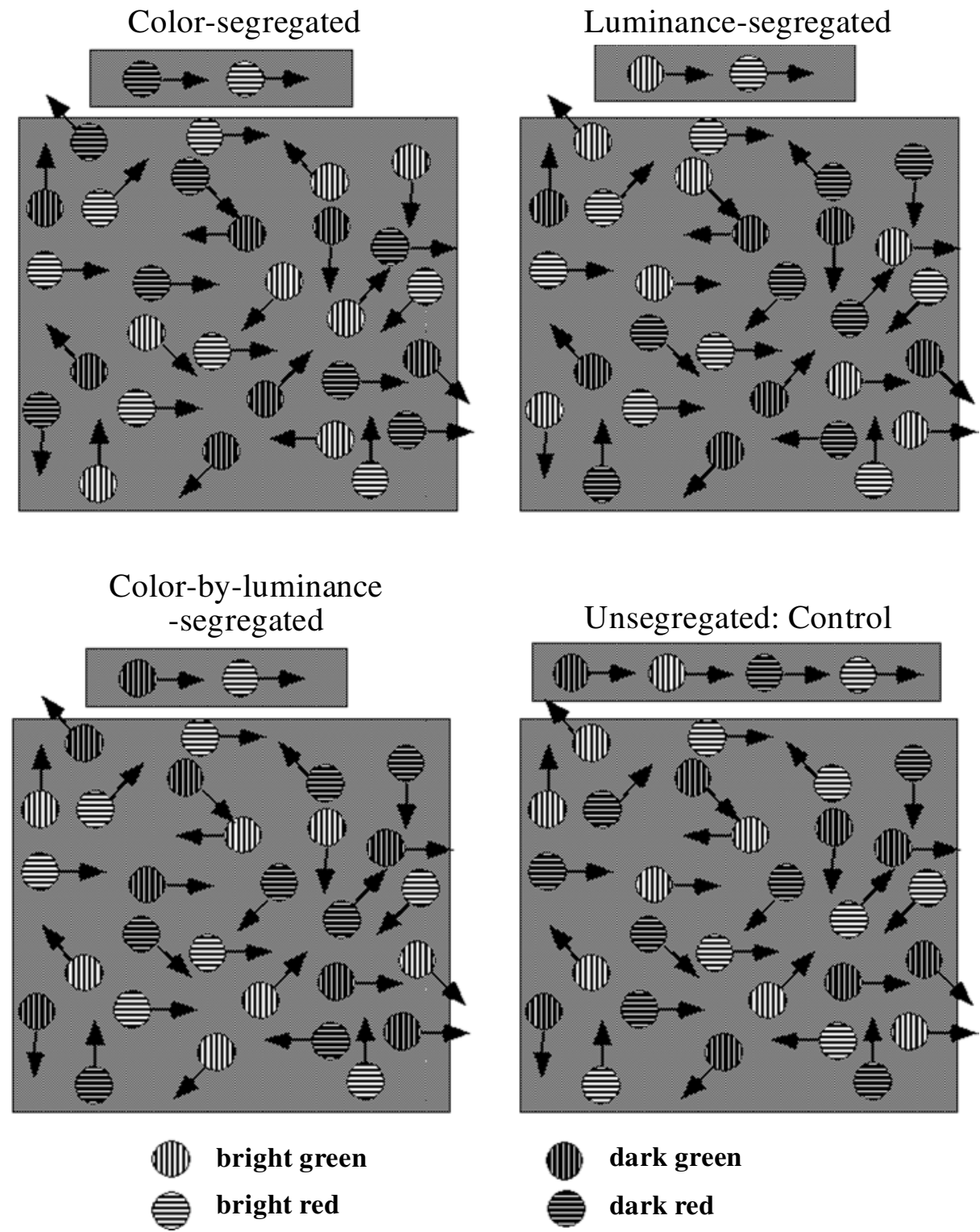

Figure 3. Schematic diagram of the stimulus used to measure coherent-motion direction discrimination in the absence of static form cues to the target. Equal numbers of the four types of dots (bright red, dark red, bright green, and dark green) were present in all the conditions. The dots in the smaller boxes above each panel indicate the target dots. Performance was measured as a function of the proportion of target dots. Because all the dots were present in equal numbers, only when the target strength reached $50 \%$ in the segregated condition was there complete segregation of target and distractor along the chosen dimension.

$50 \%$ contrast with respect to $M$ ), and $\sigma$ was the space constant of $0.076^{\circ}$. The function was clipped at a diameter of $0.34^{\circ}$. Eight types of dots were generated by combining modulations of the red and green guns in various ways, as illustrated in Figure 4. The eight types of dots were red, green, bright yellow, dark yellow, bright red, bright green, dark red, and dark green. The background was yellow-the mean of red and green. The positions of the dots were random, and when two dots overlapped, their red and green modulations (although not dc levels) were separately added. This was an important manipulation resulting in an individual dot's color and luminance contrast being added separately into the final image. Thus, for example, a bright red dot falling exactly on a dark green dot would form a uniform mid-yellow dot that was the same as the background. The manipulation was achieved by creating two independent color look-up tables (CLUTs), one for the dark red and bright green Gaussians, the other for the dark green and bright red Gaussians. A stimulus consisted of 10 apparent-motion frames. Each apparent-motion frame itself consisted of four monitor frames. The first pair of monitor frames displayed two separately generated stimuli, which alternated with the two CLUTs. The second pair of monitor frames repeated the first pair. For example, if the first motion frame consisted of dark red and bright green Gaussians, the second frame consisted of dark green and bright red Gaussians. The third and fourth frames were a repetition of the first and the second 


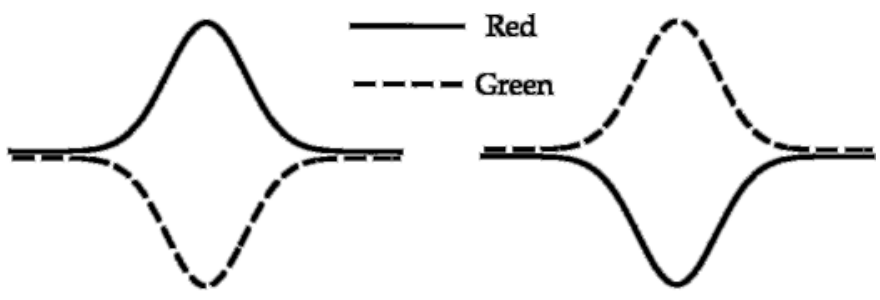

Red (isoluminant)

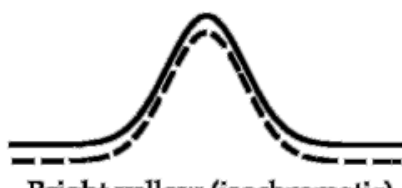

Bright yellow (isochromatic)

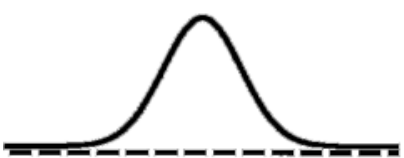

Bright red

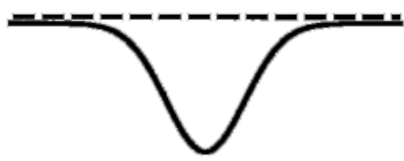

Dark red
Green (isoluminant)

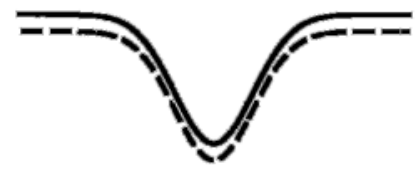

Dark yellow (isochromatic)

Figure 4. Schematic luminance profiles of the Gaussian dots employed, in terms of their red (solid line) and green (dotted line) phosphor modulations. The flat solid and dotted lines indicate the levels of the red and the green luminance, respectively, that produced the yellow background.

frames, and a series of these four motion frames constructed an apparent-motion frame. Thus, each apparent-motion frame was displayed for $33.3 \mathrm{msec}$.

Motion stimuli. Up to 100 dots were drawn in each stimulus frame within a $6.8^{\circ} \times 6.8^{\circ}$ square window. During the presentation of a stimulus, target dots all moved coherently either left or right, whereas distractor dots moved incoherently in random directions. In all but the experiments in which the target and the distractors were completely segregated (Experiments 3 and 4), the displacement size was $0.1 \mathrm{deg} /$ frame. Because each apparent-motion frame was presented for $33.3 \mathrm{msec}$ with no between-frame interval, this yielded a dot velocity of $3 \mathrm{deg} / \mathrm{sec}$. In the experiment in which the target and the distractors were completely segregated, the dots were shifted by $0.034 \mathrm{deg} / \mathrm{frame}$, resulting in a velocity of $1 \mathrm{deg} / \mathrm{sec}$. The lifetime of each dot was limited to two apparent-motion frames, to minimize the possibility that subjects would track an individual element. The total time for each stimulus presentation was $333 \mathrm{msec}$. Leftward motion was displayed by running the animation forward, and rightward motion was displayed by running the animation backward. The starting frame in a sequence was randomly chosen at every trial. The strength of the motion target was manipulated by varying the proportion of coherently moving dots.

\section{Procedure}

Before each session, the subjects adapted to a blank yellow screen for $1 \mathrm{~min}$. A fixation cross was provided between stimulus presentations, and the subjects were requested not to look away.
Each trial was initiated by the previous trial's response, a keyboard press that indicated the perceived coherent motion direction. An incorrect response was signaled by a brief tone. Before each experiment, the subjects were given sufficient practice to achieve near asymptotic levels of performance. The subjects reached saturated steady-state performance after 200 trials. Generally, different experimental conditions within an experiment were always presented in random order. Specifically, in Experiments 1 and 2, the method of constant stimuli was used to measure the discrimination threshold: From trial to trial, stimuli having different target strengths were presented randomly.

\section{Data Analysis}

For each condition, the proportion of correct responses $p$ was calculated for each target strength $c$ (the proportion of coherently moving dots). Psychometric functions were fitted using the following formula:

$$
p=1-0.5 \exp \left[-(c / \alpha)^{\beta}\right] .
$$

The Levenberg-Marquardt algorithm (Marquardt, 1963; Press, Teukolsky, Vetterling, \& Flannery, 1992) was used to fit the threshold at the $82 \%^{1}$ correct level and slope $\beta$ so that the chi-square was minimized. Goodness of fit at the $p<.05$ level was tested using chisquares, and every fit was found to be satisfactory. For each subject, we performed a statistical test, based on the method employed by Britten, Shadlen, Newsome, and Movshon (1992), to determine whether thresholds for any two conditions were significantly differ- 

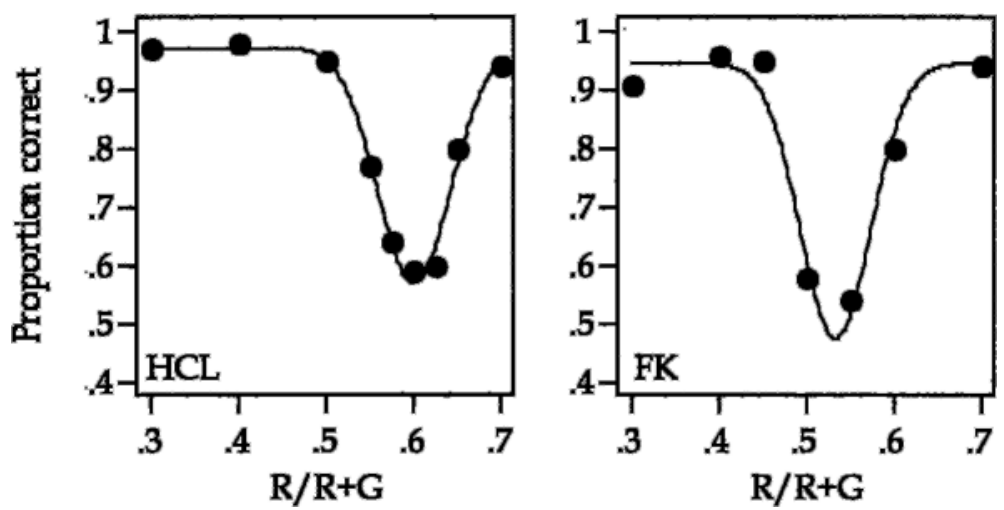

Figure 5. Proportion correct as a function of the $R /(R+G)$ mean luminance ratio for stimuli made up of red and green dots. Target strength (proportion of coherently moving dots) was fixed at $\mathbf{4 0 \%}$.

ent. We compared the chi-square values obtained from fitting the data in two ways. In the first, psychometric functions were fitted individually to the two data sets, and the resulting pair of chi-squares was summed. In the second, psychometric functions were fitted to the two data sets, with the constraint that the thresholds were equal, and again the two resulting chi-squares were summed. If the difference between the summed chi-squares obtained from the two methods $\left(\chi_{\text {diff }}^{2}\right)$ exceeded the criterion value $(d f=1, p<.05$ in chi-square distribution), we concluded that the thresholds for the two conditions were significantly different.

\section{Motion Direction Discrimination \\ as a Function of the $R /(R+G)$ Ratio}

In order to ensure that the chromatic content of the dots did not contribute additional luminance components to the stimuli, we first measured the isoluminant red-to-green mean luminance ratio, $R /(R+G)$. The $R /(R+G)$ ratio is the ratio of the red to the summed red-plus-green mean luminances (with dot contrast, defined as amplitude/mean, held constant) that produced the worst motion direction discrimination. The stimuli consisted of 100 dots, $50 \%$ red and $50 \%$ green, with $40 \%$ of the dots moving coherently. For each $R /(R$ $+G)$ value, 240 trials were conducted spread over three sessions. The results are shown in Figure 5. At low and high $R /(R+G)$ ratios, the subjects performed the task at near $100 \%$ performance, but around the middle of the range, performance collapsed to near-chance levels. The continuous function running through the data is the bestfitting Gaussian function, and the $R /(R+G)$ value producing the lowest performance was estimated from the fit. This was .6 for H.-C.L., .53 for F.K., .6 for M.K., .57 for E.O., and .52 for M.R. These isoluminant $R /(R+G)$ ratios were used throughout the rest of the study.

\section{EXPERIMENT 1 Replication of Croner and Albright (1997)}

\section{Aim and Method}

We first wanted to determine that the results of Croner and Albright (1997) were found with our stimuli. The experimental scheme was as close to Croner and Albright's (1997) as possible, the main difference being that they used plain circular dots, whereas we used Gaussian dots. We first employed two types of dots, and then extended the paradigm to four types.

Figure 2 illustrates the stimuli. In the color-segregated test stimulus, there were two types of Gaussian dots, bright red and bright green, segregated into target and distractor. The target color was randomly chosen on each trial. In the color-uniform control stimulus, all the dots were bright yellow and had twice the luminance contrast of the dots in the color-segregated condition. Setting the dots to the same luminance contrast in both conditions invites the criticism that the dots in the color-segregated condition are more salient because of their additional color contrast and that this alone produces better performance. We opted for roughly equal saliency by setting the sum of each dot's luminance and color contrast to be equal in both conditions, as measured in terms of gun modulation. The proportion of coherently moving dots was varied in both the color-segregated and the color-uniform conditions, between $0.45 \%$ and $40 \%$. For each target strength, 200 trials were conducted over five separate sessions, and the overall percentage correct was calculated.

The same principle underlay the construction of stimuli with four types of dots. In the color-segregated condition, the target was either an equal number of bright red and dark red dots, with the distractor an equal number of bright green and dark green dots, or vice versa. In the color-uniform condition, an equal number of bright yellow and dark yellow dots was present.

\section{Results}

Figure 6 shows the results for the two-dot stimuli. The dotted and continuous lines are the fitted psychometric functions to the color-segregated and color-uniform conditions, respectively. As was expected, performance in both conditions improved with target strength, and performance was better in the color-segregated condition. Thresholds for the color-segregated condition were $2.96 \%$ for H.-C.L. and $3.26 \%$ for F.K., and those for the color-uniform condition were $14.58 \%$ for H.-C.L. and $17.59 \%$ for F.K. The difference in thresholds between the two conditions is highly significant $\left[\chi_{\text {diff }}^{2}(1)=49.53\right.$, $p<.05$ for H.-C.L.; $\chi_{\text {diff }}^{2}(1)=23.84, p<.05$ for F.K.]. These results essentially replicate the pattern found by Croner and Albright (1997), who found thresholds varying between $0.5 \%$ and $1.8 \%$ for the color-segregated (their "hetero-chromatic") condition and between $3.1 \%$ and $6.2 \%$ for the color-uniform (their "homo-chromatic") condition.

The results for the four-dot stimuli are shown in Figure 7. Color-segregated thresholds were about one third of the color uniform thresholds for both subjects $\left[\chi_{\text {diff }}^{2}(1)=\right.$ 

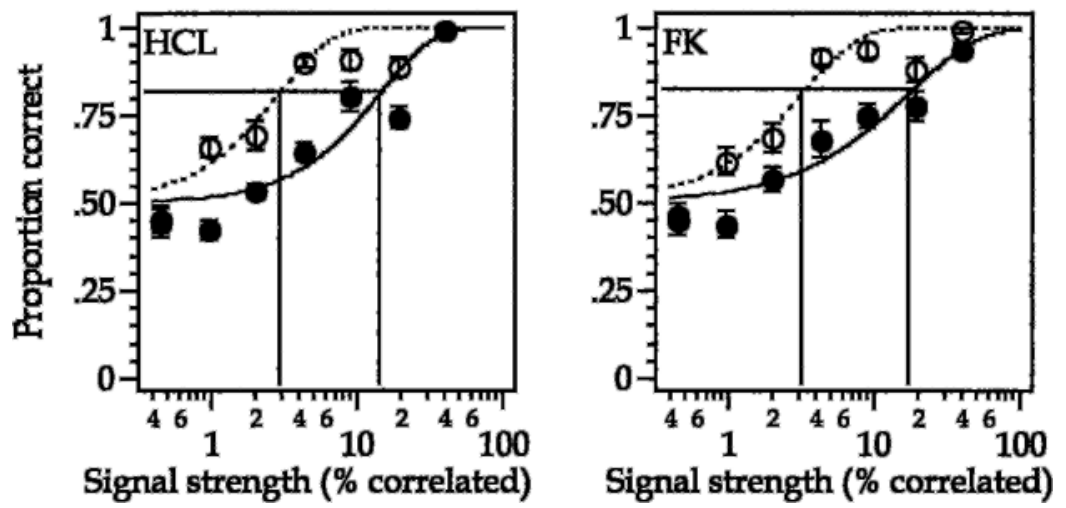

Figure 6. Results for Experiment 1 (replication of Croner \& Albright, 1997) using two types of dots (bright red and bright green) in the color-segregated condition and one type (bright yellow) in the color-uniform condition, as is illustrated in Figure 2. Proportion correct is plotted against the proportion of target dots. Open circles are for the color-segregated condition, and filled circles are for the color-uniform condition. The dotted and continuous lines are Weibull fits to the data. The intersection of the horizontal line to the fitted curves indicates the threshold target strength at the $82 \%$ correct level. The number of trials for each data point was 200 spread over five sessions for both subjects. Error bars indicate standard errors of the percentage of correct scores over the five sessions. The fitted parameters are as follows, with $\alpha_{\text {seg }}$ and $\alpha_{\text {unif }}$ thresholds for the color-segregated and color-uniform conditions, and $\beta_{\text {seg }}^{\text {seg and }}$ $\beta_{\text {unif }}$ for slopes: H.-C.L., $\alpha_{\text {seg }}=2.961, \beta_{\text {seg }}=1.225, \alpha_{\text {unif }}=14.580, \beta_{\text {unif }}=1.208$; F.K., $\alpha_{\text {seg }}=3.264, \beta_{\text {seg }}=1.119, \alpha_{\text {unif }}=17.592, \beta_{\text {unif }}=0.924$.

$18.45, p<.05$ for H.-C.L.; $\chi_{\text {diff }}^{2}(1)=4.78, p<.05$ for F.K.], thus extending the replication of Croner and Albright (1997) to stimuli consisting of four, as opposed to just two types of dot.

\section{EXPERIMENT 2 \\ Motion Discrimination Without Static Form Cues and With Incomplete Segregation of Target and Distractor}

\section{Overall Aim}

The results of Experiment 1 are readily explained in terms of the presence of static form cues. The target dots, although "camouflaged" in the color-uniform condition, tend to pop out in the color-segregated condition, since they are a minority, providing a static form cue to the target. What if no static form cues were present? Does the visual system automatically prefilter the color-segregated stimulus into different color maps and process the motion properties of each map independently, resulting in better performance than with the unsegregated comparison stimulus? To test this possibility we first employed a paradigm similar to that of Edwards and Badcock $(1994,1996)$; Figure 3 illustrates the stimuli. Unlike Edwards and Badcock, we used the same composition of dot types in both test (segregated) and comparison (unsegregated) conditions and, in the former condition, randomized on every trial which dots were target and which distractors. This ensured that any differences in the saliencies of the different dot types could not systematically favor either target or distractor and that there were no differences between the test and the comparison conditions in overall mean luminance or chromaticity. Moreover, by randomizing on each trial of the segregated condition which dot type contained the target, we eliminated the possibility of any prior selective attention to the target. A stationary frame of any of the stimuli in this experiment would provide no clue as to which dot type contained the target. Is performance nevertheless superior in the segregated condition?

\section{Experiment 2A \\ Incomplete Segregation With Four Dot Types Method \\ The conditions were (refer to Figure 3): (1) color-segregat ed (bright red and dark red vs. bright green and dark green), (2) luminance- segregated (bright red and bright green vs. dark red and dark green), (3) color-by-luminance-segregated (bright red and dark green vs. dark red and bright green), and (4) unsegregated (bright red, dark red, bright green, and dark green for both target and distractor). It is important to note that by keeping the numbers of each dot type the same, the degree to which the target and the distractors were segregated by dot type depended on target strength. Consider, for example, the situation in which $25 \%$ of the dots in the stimulus were}




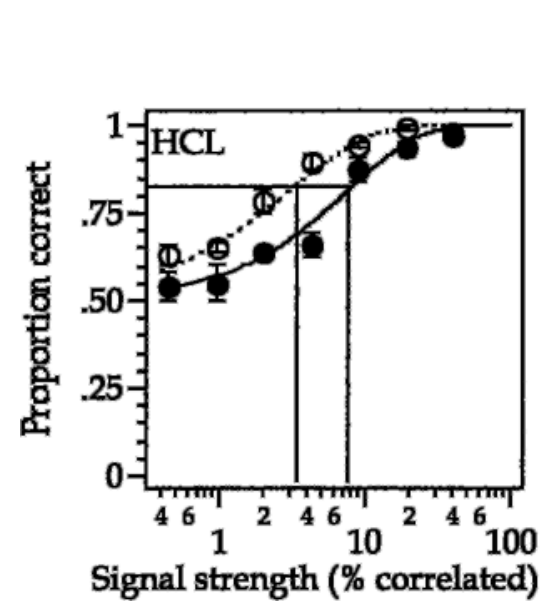

\section{O color-segregated color-uniform}

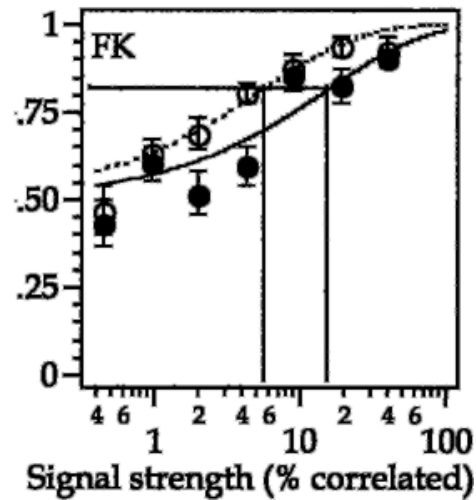

Figure 7. Results for Experiment 1 using four types of dots (bright red, dark red, bright green, and dark green) in the color-segregated condition and two types of dots (bright yellow and dark yellow) in the color-uniform condition. All symbols are the same as those in Figure 6. Fitted parameters are as follows: H.-C.L., $\alpha_{\text {seg }}=3.044, \beta_{\text {seg }}=$ 0.796, $\alpha_{\text {unif }}=7.712, \beta_{\text {unif }}=0.904 ;$ F.K., $\alpha_{\text {seg }}=5.608, \beta_{\text {seg }}=0.650, \alpha_{\text {unif }}=15.616, \beta_{\text {unif }}=$ 0.665 .

the target and $75 \%$ distractors. In the color-segregated condition (see Table 1), this meant that although all the target dots were, say, red (bright and dark), one third of the distractor dots were also red. Thus, only in the $50 \%$ target strength color-segregated condition were red and green dots completely segregated into target and distractors. In the comparison, unsegregated condition (Condition 4), red and green dots were equally distributed between target and distractors.

\section{Results}

The results are shown in Figure 8. Discrimination of the direction of coherent motion was generally better in the segregated conditions than in the unsegregated condition, but the differences were small and within error range. For both subjects, the biggest difference was between the luminance-segregated and the luminance-unsegregated conditions, in which thresholds were $9.41 \%$ and $12.15 \%$ for H.-C.L. and $16.29 \%$ and $23.38 \%$ for F.K., respectively. For both subjects, however, these differences were not significant $\left[\chi_{\text {diff }}^{2}(1)=2.24, p>.05\right.$ for H.-C.L.; $\chi_{\text {diff }}^{2}(1)=$ $1.47, p>.05$ for F.K.].

\section{Experiment 2B}

\section{Incomplete Segregation With Two Dot Types}

\section{Aim and Method}

In some of the conditions of the previous experiment, we found a small, albeit nonsignificant, superiority of the segregated over the unsegregated conditions (see Figure 8). It is possible that employing four, as opposed to two types of dots reduced the potential benefits of segregation. If only two types of dots were used, would the effect become signif icant? Conversely, the small superiority of the segregated condition in the previous experiment may have resulted from the subjects' attending more strenuously to this condition than to the comparison, unsegregated condition. Both these possibilities were controlled in the present experiment by using a stimulus containing only two types of dots and randomly mixing both segregated and unsegregated conditions within the same session.

The conditions were (1) the color-segregated (dark red vs. dark green or bright red vs. bright green) and color-unsegregated (dark red and dark green or bright red and bright green) conditions, (2) the luminance-segregated (dark red vs. bright red or dark green vs. bright green) and luminance-unsegregated (dark red and bright red or dark green and bright green) conditions, and (3) the color-

Table 1

Distribution of Target and Distractor Dots in Color-Segregated Condition in Experiment 2A

\begin{tabular}{|c|c|c|c|c|c|c|c|c|}
\hline $\begin{array}{c}\text { Target } \\
\text { Strength }(\%)\end{array}$ & $\begin{array}{c}\% \text { of } \\
\text { Distractor Dots }\end{array}$ & $\begin{array}{l}\text { Target } \\
\text { Color }\end{array}$ & $\begin{array}{c}\% \text { of } \\
\text { Red Dots }\end{array}$ & $\begin{array}{c}\% \text { of } \\
\text { Green Dots }\end{array}$ & $\begin{array}{l}\% \text { of Red } \\
\text { and Target }\end{array}$ & $\begin{array}{c}\% \text { of Green } \\
\text { and Target }\end{array}$ & $\begin{array}{c}\% \text { of Red } \\
\text { and Distractor }\end{array}$ & $\begin{array}{c}\% \text { of Green } \\
\text { and Distractor }\end{array}$ \\
\hline 25 & 75 & red & 50 & 50 & 25 & 0 & 25 & 50 \\
\hline 25 & 75 & green & 50 & 50 & 0 & 25 & 50 & 25 \\
\hline 35 & 65 & red & 50 & 50 & 35 & 0 & 15 & 50 \\
\hline 35 & 65 & green & 50 & 50 & 0 & 35 & 50 & 15 \\
\hline 50 & 50 & red & 50 & 50 & 50 & 0 & 0 & 50 \\
\hline 50 & 50 & green & 50 & 50 & 0 & 50 & 50 & 0 \\
\hline
\end{tabular}

Note-The stimuli were made up of the same proportion of red and green dots. When the target strength was $25 \%$ and target color was red, $50 \%$ of the dots were green and distractors and $25 \%$ of the dots were red and distractors. It should be noted that the target and the distractor were not completely segregated by color except when the target strength was $50 \%$. 
(A)
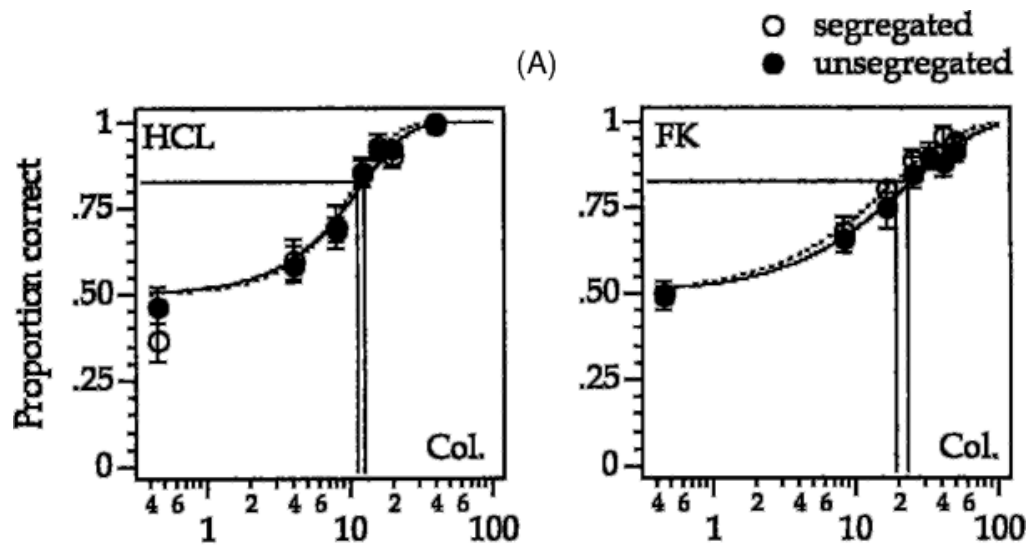

(B)
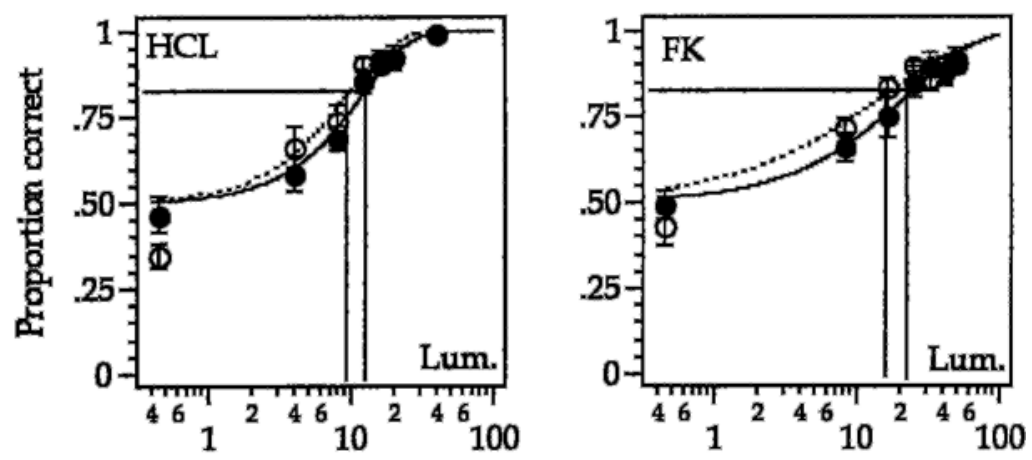

(C)
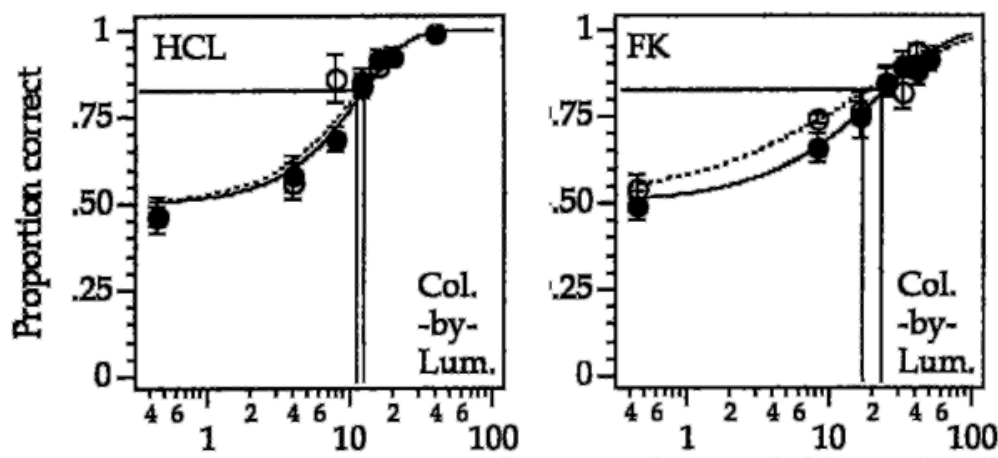

Signal strength (\% correalted)

Signal strength (\% correlated)

Figure 8. Results for Experiment 2A (absence of static form cues but incomplete segregation of target and distractor), which used four types of dots (bright red, dark red, bright green, and dark green). Thresholds and slopes are as follows ( $\alpha_{\text {unseg }}$ and $\beta_{\text {unseg }}$ indicate the threshold and slope for the unsegregated condition). (A) Color: H.-C.L., $\alpha_{\text {seg }}=11.179, \beta_{\text {seg }}=1.513, \alpha_{\text {unseg }}=12.150, \beta_{\text {unseg }}=1.326 ;$ F.K., $\alpha_{\text {seg }}=18.143$, $\beta_{\text {seg }}=0.905, \alpha_{\text {unseg }}=\mathbf{2 3 . 3 8 1}, \beta_{\text {unseg }}=0.900$. (B) Luminance: H.C.L., $\alpha_{\text {seg }}=9.412, \beta_{\text {seg }}=$ $1.258, \alpha_{\text {unseg }}=12.150, \beta_{\text {unseg }}=1.326 ;$ F.K. $, \alpha_{\text {seg }}=16.291, \beta_{\text {seg }}=0.6957, \alpha_{\text {unseg }}=23.381$, $\beta_{\text {unseg }}=0.900$. (C) Color-by-luminance: H.-C.L., $\alpha_{\text {seg }}=11.346, \beta_{\text {seg }}=1.198, \alpha_{\text {unseg }}=$ 12.150, $\beta_{\text {unseg }}=1.326$; F.K., $\alpha_{\text {seg }}=17.253, \beta_{\text {seg }}=0.598, \alpha_{\text {unseg }}=23.381, \beta_{\text {unseg }}=0.900$.

plus-luminance-s egregated (bright red vs. dark green or bright green vs. dark red) and color-plus-luminance-unsegregated (bright red and dark green or bright green and dark red) conditions.

\section{Results}

The results are shown in Figure 9. Generally, decreasing the number of dot types from four to two resulted in a small overall improvement in performance. However, it did not produce significantly better performance in the segregated than in the unsegregated conditions. We conclude that irrespective of whether four or two types of dots are used, and irrespective of whether there is prior knowledge as to the segregated/unsegregated nature of the stimulus, there is no significant difference between the 
segregated and the unsegregated conditions in the present paradigm.

\section{EXPERIMENT 3 \\ Motion Discrimination Without Static Form Cues but With Complete Segregation of Target and Distractor}

\section{Overall Aim}

One reason for the lack of a significant effect of segregation in the previous experiment, as well as in the Edwards and Badcock $(1994,1996)$ studies described earlier, is that for all except the $50 \%$ target strength condition, the target and the distractors were never fully segregated by dot type. The potentially beneficial effects of segregation may simply not have become manifest. We therefore decided to measure coherent-motion discrimination thresholds in the absence of static form cues, but this time with target and distractors fully segregated by dot type.

\section{Experiment 3A}

Complete Segregation of Target and Distractor

With 50\% Target Strength and Four Dot Types

\section{Aim and Method}

Because performance was near $100 \%$ in the previous experiments for the $50 \%$ target strength, we reduced performance by decreasing the displacement size from $0.1 \mathrm{deg} /$ frame to $0.034 \mathrm{deg} /$ frame (1 $\mathrm{pixel} /$ frame). It is important to reiterate that even though the target strength was $50 \%$ and thus fully differentiated by dot type from the distractor in the segregated condition, no static form cues to the target were present. If an observer were presented with a stationary frame of either the segregated or the unsegregated condition, he/she would be unable to tell which dots were the target and which the distractors. The four conditions were (1) color-segreg ated, (2) luminancesegregated, (3) color-by-luminance-segregated, and (4) unsegregated conditions.

\section{Results}

Figure 10 shows the results. Each data point represents performance over 200 trials. Complete segregation by color, luminance polarity, or both dimensions did not significantly improve performance, as compared with the unsegregated condition $[F(3,9)=1.165, p=.3757]$. Table 2 provides details of all the pairwise comparisons. The observers achieved near-100\% performance when the distractor dots were removed altogether, implying that the distractor dots deteriorated performance even when differently colored from the target dots.

\section{Experiment 3B}

Complete Segregation of Target and Distractor

With $50 \%$ Target Strength and Two Dot Types

\section{Aim and Method}

As in Experiment 2A, there was a small, albeit nonsignificant, superiority of the segregated condition over the unsegregated condition. Therefore, we again decided to repeat Experiment $3 \mathrm{~A}$, using just two dot types. Four types of the color-segregated condition were used: (1) dark red target-dark green distractor, (2) dark green target-dark red distractor, (3) bright red target-bright green distractor, and (4) bright green target-bright green distractor. The corresponding unsegregated conditons were (1) dark red, dark green target-dark red, dark green distractor and (2) bright red, bright green target-bright red, bright green distractor.

\section{Results}

The results for the four types of the color-segregated condition and the two types of the unsegregated condition were averaged for each subject. The proportion correct was, on average, .702 for the color-segregated condition and .758 for the unsegregated condition, but the difference was not significant $[F(1,4)=4.146, p=.1114]$. Without the distractor dots, the subjects' performances were almost perfect, again implying that the distractor significantly deteriorated performance.

\section{Experiment 3C}

\section{Complete Segregation of Target and Distractor With $25 \%$ Target Strength and Four Dot Types}

\section{Aim and Method}

In this experiment, all four types of dots were employed in equal numbers, but the target was set to $25 \%$. In the segregated condition, only one type of dot was the target, the other was the distractor, and the dot chosen to be the target was randomly selected on each trial. In the unsegregated condition, the target consisted of equal numbers of all four dots. Segregated and unsegregated conditions were randomly mixed within a session.

\section{Results}

The data were pooled and averaged over four types of the segregated condition and over the corresponding unsegregated conditions. The proportion of the correct performances were .705 for the segregated condition and .736 for the unsegregated condition, but the difference was not significant $[F(1,4)=1.625, p=.2714]$. The subjects' performances were almost perfect when the random distractor were removed from the stimuli, implying that the addition of a random distractor significantly deteriorated the subjects' performances.

\section{EXPERIMENT 4 \\ Effect of Selective Attention to the Target}

\section{Overall Aim}

In all the above experiments, the subjects did not know on each trial which types of $\operatorname{dot}(\mathrm{s})$ were the target. What if they did know? Would performance in the segregated conditions now be superior? In the following experiments, we tested this possibility. Sessions in which the subject knew the target dot type were always mixed with sessions in which they did not.

\section{Experiment 4A \\ Selective Attention With Complete Segregation of Target and Distractor, $50 \%$ Target Strength, and Two Dot Types}

\section{Aim and Method}

In this experiment, we employed stimuli in which the target and the distractor were completely segregated by color (as in Experiment 3). Each stimulus consisted of two types of dot. The two types of the color-segregated condition employed (1) dark red and dark green dots and (2) bright red and bright green dots. The corre- 
(A)

O segregated

- unsegregated
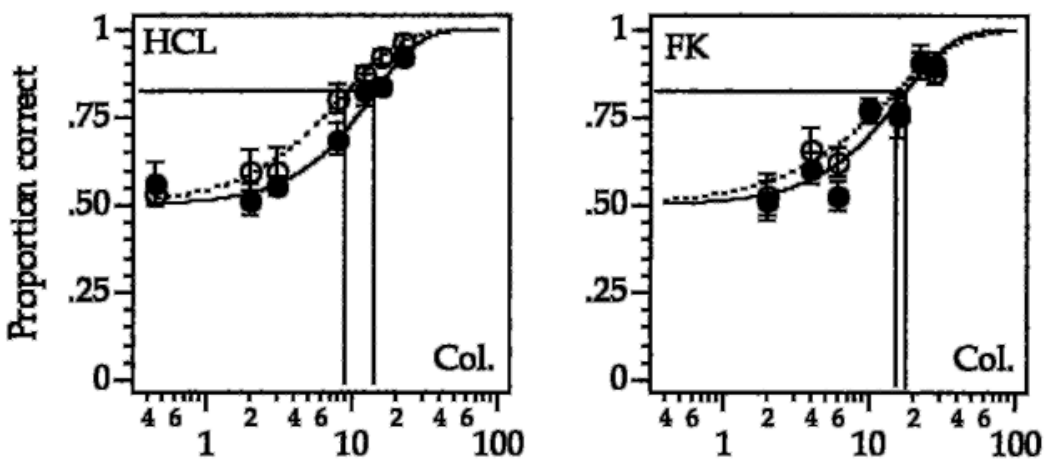

(B)
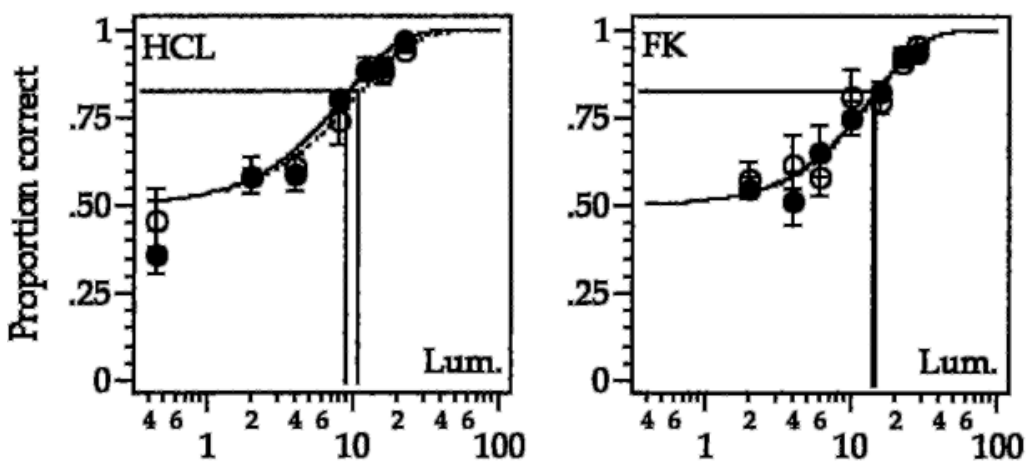

(C)
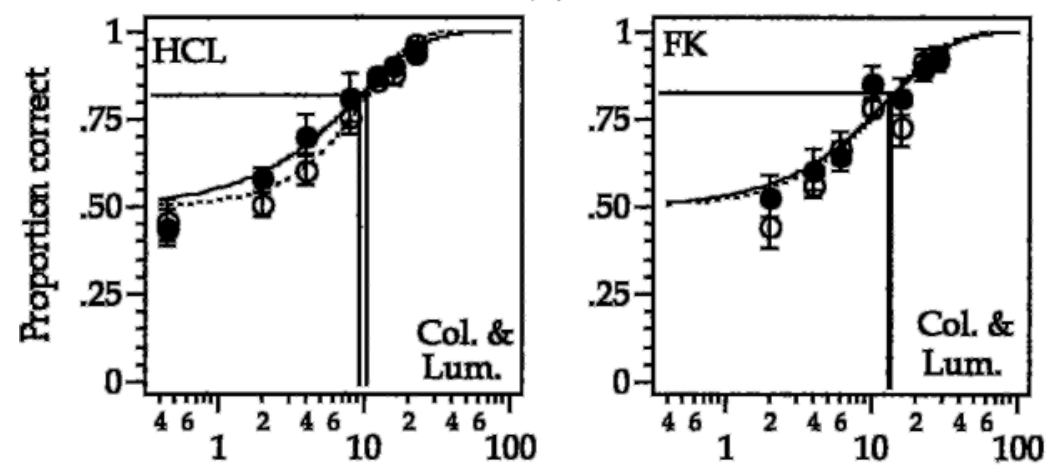

Signal strength (\% correlated) Signal strength (\% correlated)

Figure 9. Results for Experiment 2B (absence of static form cues but incomplete segregation of target and distractor), which used two ty pes of dots. (A) Color: H.-C.L., $\alpha_{\text {seg }}=$ 8.892, $\beta_{\text {seg }}=1.083, \alpha_{\text {unseg }}=14.059, \beta_{\text {unseg }}=1.339 ;$ F.K., $\alpha_{\text {seg }}=14.779, \beta_{\text {seg }}=0.963, \alpha_{\text {unseg }}=$ 17.106, $\beta_{\text {unseg }}=1.257$. (B) Luminance: H.-C.L., $\alpha_{\text {seg }}=10.847, \beta_{\text {seg }}=1.116, \alpha_{\text {unseg }}=8.950$, $\beta_{\text {unseg }}=1.197$; F.K., $\alpha_{\text {seg }}=14.839, \beta_{\text {seg }}=1.315, \alpha_{\text {unseg }}=14.455, \beta_{\text {unseg }}=1.290$. (C) Colorplus-luminance: H.-C.L., $\alpha_{\text {seg }}=10.434, \beta_{\text {seg }}=1.381, \alpha_{\text {unseg }}=9.232, \beta_{\text {unseg }}=0.985$; F.K., $\alpha_{\text {seg }}=13.589, \beta_{\text {seg }}=1.130, \alpha_{\text {unseg }}=13.732, \beta_{\text {unseg }}=1.023$. 


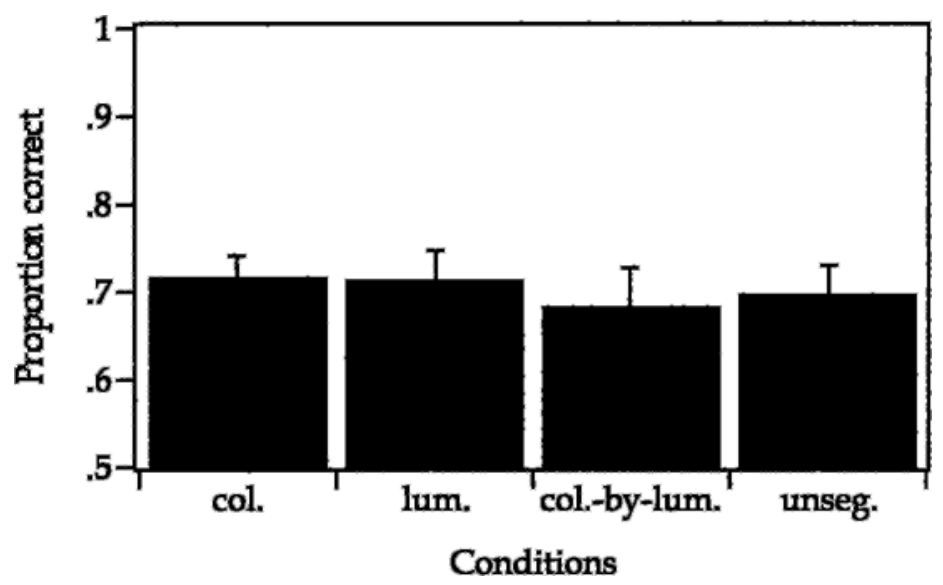

Figure 10. Results for Experiment 3A (absence of static form cues and complete segregation of target and distractor). Stimuli consisted of four types of dots (bright red, dark red, bright green, and dark green). Target strength was fixed at $50 \%$, resulting in complete segregation of target and distractor by dot type in the segregated condition. Proportion of correct scores were the following: H.-C.L., color $=.797$, luminance $=.7929$, color-byluminance $=.8237$, unsegregated $=.8085 ;$ F.K., color $=.7408$, luminance $=$ .6559 , color-by-luminance $=\mathbf{. 6 8 8 5}$, unsegregated $=.7679$.

sponding unsegregated comparison conditions used the same types of dots divided equally between target and distractor. No-distractor conditions were also included.

\section{Results}

For each subject, the responses in the various types of the segregated condition were averaged. The proportion correct across all 5 subjects was .792 for the segregatedwith-attention condition and .703 for the unsegregated condition. This difference was marginally significant $[F(1,4)=5.909, p=.0719]$. Whereas both authors showed a significant attention effect, the naive subjects did not (see Table 3). Performance was almost perfect in all the no-distractor conditions.

\section{Experiment 4B \\ Selective Attention With Complete Segregation of Target and Distractor, $25 \%$ Target Strength, and Four Dot Types}

\section{Aim and Method}

It is possible that the effects of selective attention best manifest themselves when the target dots are in a minority. Therefore, we used the same stimuli as those employed in Experiment 3C, in which four dot types were employed (bright red, bright green, dark red, and dark green) and the target consisted of $25 \%$ of the dots. The difference in this experiment, however, was that the subject knew the type of target dot in every segregated condition and was required to selectively attend to it. In the unsegregated condition, all four dot types were present in both the target and the distractor in equal proportions.

\section{Results}

The proportion correct was .847 for the segregatedplus-attention condition and .763 for the unsegregated condition. The difference was significant $[F(1,4)=23.691$, $p=.0082]$. The subjects' performances were again almost perfect in the no-distractor condition.

\section{GENERAL DISCUSSION}

The primary goal of this study was to determine whether motion-direction discrimination in distractors benefited from segregation of target and distractor by color, luminance polarity, or both, under conditions in which static form cues were eliminated. Our results suggest that without prior knowledge of the target dot type, segregation by color/luminance does not benefit performance in the absence of static form cues. We eliminated static form cues by setting equal the numbers of the different dot types and randomizing, on each trial, which dot type(s) were target and which distractor; under these conditions, no superiority was observed in any of our segregated conditions.

Table 2

Statistical Details From the Comparison of the Segregated and Unsegregated Conditions of the Experiment Where Target and Distractor Were Completely Segregated by Color/Luminance Polarity in the Segregated Conditions

\begin{tabular}{ccc}
\hline $\begin{array}{c}\text { Color-Segregated } \\
\text { Versus Unsegregated }\end{array}$ & $\begin{array}{c}\text { Luminance-Segregated } \\
\text { Versus Unsegregated }\end{array}$ & $\begin{array}{c}\text { Color-by-Luminance } \\
\text { Segregated Versus Unsegregated }\end{array}$ \\
\hline$F(1,3)=0.848, p=.3811$ & $F(1,3)=0.613, p=.4538$ & $F(1,3)=0.526, p=.4868$ \\
\hline
\end{tabular}


Table 3

Mean Proportion of Correct Responses for Each Subject in Segregated/Unsegregated Conditions in Experiment 4A

\begin{tabular}{lcc}
\hline Subject & Segregated Condition & Unsegregated Condition \\
\hline F.K. & .8372 & .7113 \\
H.-C.L. & .9764 & .7731 \\
M.K. & .662 & .6768 \\
E.O. & .7552 & .6823 \\
M.R. & .7291 & .6374 \\
\hline
\end{tabular}

Note-Both F.K. and H.C.L. are the authors, and the others are naive subjects.

The only circumstance in which we found that segregation by color/luminance improved performance in the absence of static form cues was when the target consisted of one of four types of dots and there was prior knowledge of the target dot type.

Our results confirm previous findings of both Edwards and Badcock $(1994,1996)$ and Croner and Albright (1997) but, importantly, qualify and extend their results. In the case of the Edwards and Badcock studies, we confirmed that motion discrimination was not facilitated when target and distractors were segregated by dot type, when the two dot types were present in equal numbers. In addition we showed that this result could not be due to either (1) differences in the saliencies of the two dot types or (2) incomplete segregation of the target and the distractor by dot type. In the case of the Croner and Albright (1997) study, we confirmed that, under conditions in which the target dots were a minority and fully segregated from the distractors, motion discrimination was superior when target and distractors differed in dot type.

Croner and Albright (1997) suggested that their results could be due to either preattentive or selective-attentive segmentation of the differently colored target dots. Recently, Snowden and Edmunds (1999), employing a paradigm similar to that of Croner and Albright, showed that if the stimuli were shown peripherally, or if their duration was very brief, the positive color-segregation effect disappeared. Snowden and Edmunds argued that this supports the selective-attentionalexplanation. The results from our selective attention experiments are consistent with this conclusion. However, our results add the important caveat that the positive segregation effect does not necessarily require that the target dots (being only a few and of a different color) "grab" the attention of the observer. Provided the target dots are small in number (in our experiment, $25 \%$ ), prior knowledge of and selective attention to the target dots can be sufficient to produce a positive segregation effect; it does not occur only when they pop out in a static view of the stimulus.

What is the mechanism by which these attention effects operate? Recent fMRI studies have isolated specific brain areas for attentive tracking in high-level apparent-motion perception (Culham et al., 1998), and there is psychophysical evidence for a specific tracking mechanism in motion perception (Pylyshyn \& Storm, 1988). Did our sub- jects track the target dots to which their attention was directed? Although we cannot rule out this possibility, it seems unlikely. In our study, each individual element lasted, at most, two frames ( $66 \mathrm{msec}$ ) and always appeared in a random position. If the beneficial effects of selective attention in our experiments were not mediated by tracking, then, how were they mediated? One possibility is that attention in the global motion paradigm operates rather like a filter, allowing the visual system to selectively process the motion properties of a limited number of objects with a common color/luminance; in this view, selective attention simply filters out the distractors.

How do our results relate to traditional visual search paradigms, in which single targets are presented among an array of distractors? The stimulus make-up in our attention experiment (Experiment 4 ) has certain superficial similarities to the conjunction case in visual search (Berger \& McLeod, 1996; Driver \& McLeod, 1992; McLeod, Driver, \& Crisp, 1988; McLeod, Driver, Dienes, \& Crisp, 1991; Müller \& Found, 1996; Nakayama \& Silverman, 1986), since our target was defined as a conjunction of motion and color/luminance. However, our task was quite different. For example, in the experiment of Nakayama and Silverman (1986), subjects searched for a target defined by a unique color and a unique motion direction. In our Experiment 4, the subjects reported the direction of motion of a target that they knew to be of a particular color; no conjunction was sought.

In the experiments in which we found superiority in the segregated condition over the unsegregated condition, we conclude that the mechanisms that process global motion direction do not automatically prefilter the image into separate color/luminance maps and process their motion properties in parallel. Only with static form cues, or attention to the known target color, does direction-of-motion discrimination appear to benefit from segregation. This is, arguably, consistent with the idea that, as a general rule, motion-integrative mechanisms are form-cue invariant (Albright, 1992; Stoner \& Albright, 1993).

Why do motion mechanisms fail to automatically group together features by color or luminance polarity and process the resulting color/luminance maps in parallel? One reason we can reject is that local motion detectors are not sensitive to color, luminance polarity, or combinations of the two. In the introduction, we mentioned a number of studies that have demonstrated a chromatic input to motion mechanisms. Of particular relevance is the study by Morgan and Ingle (1994), who, like us, used stimuli containing both luminance and chromatic contrast. They found that when the color contrast in a nonisoluminant red-green random-dot kinematogram was reversed between frames (i.e., the red elements became green and the green red), motion perception was impaired even though the luminance contrasts remained perfectly correlated between frames. With regard to luminance polarity, numerous studies have testified to the importance of this dimension for local motion detection (Livingstone \& Hubel, 


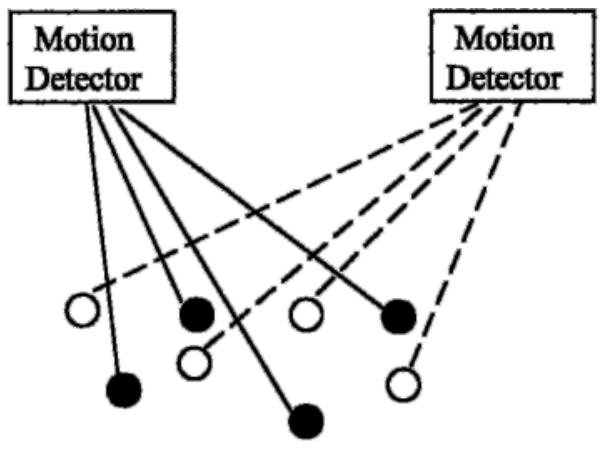

(A)

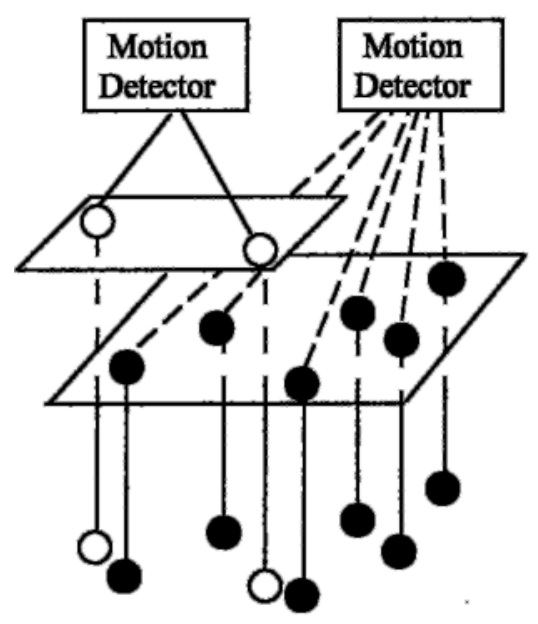

(C)

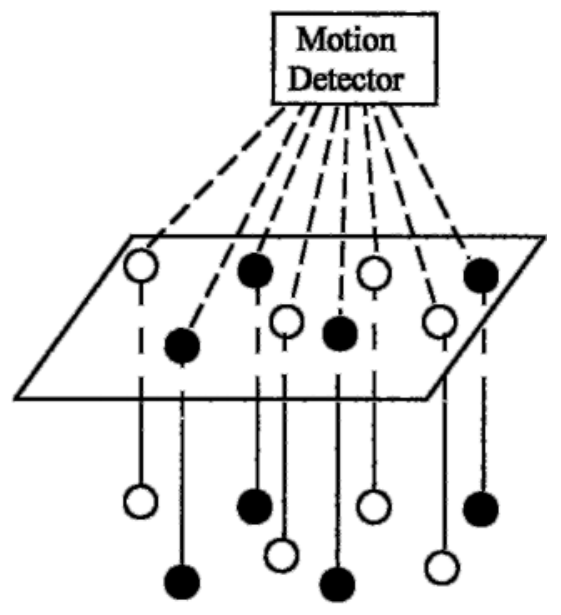

(B)

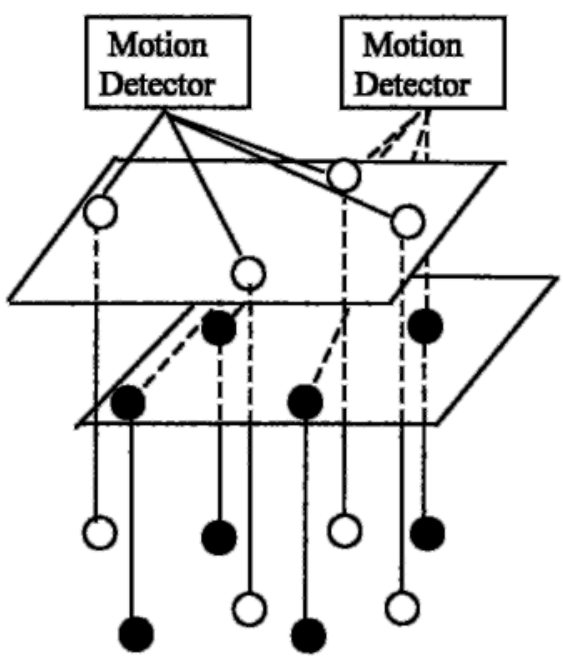

(D)

Figure 11. Four schematic diagrams show the possible relationships between form processing and motion processing. (A) The rejected model: Local features are automatically grouped by color/luminance prior to motion processing. (B) Instead, the motion system integrates local feature information regardless of color/luminance. (C) When target elements are in a minority and differ in color/luminance from the distractors, the resulting static form cue allows them to be segregated from the distractors prior to motion processing. (D) Selective attention to the known target color helps segregate the target from distractors and facilitate motion processing, even though static form cues are absent.

1988; Ramachandran \& Gregory, 1978; Schiller, Logothetis, \& Charles, 1990; Zeki \& Shipp, 1988).

A more plausible explanation for the lack of an effect of color/luminance segregation when both static form cues are absent and selective attention to the target color/ luminance is impossible comes from an examination of the perceptual needs of animals living in densely textured environments, such as forests. Would it always make sense in such circumstances for the visual system to automatically group features with similar colors/luminances prior to motion processing? Consider the case of a multi-colored animal moving through foliage. The multicoloring itself would provide an effective means of camouflage, since it would perceptually "break up" the structure of the animal. 
In this case, the most reliable feature for identifying the animal's structure would be its coherent direction of motion. If motion mechanisms automatically operated separately on the various colors making up the object, the visual system would have to integrate a number of separate color-motion maps to establish the presence of a single coherently moving object. This would be time consuming and require much neural hardware and would be less efficient than if the motion system simply ignored color and concentrated on establishing which elements were moving coherently and which were not.

The situation would be different, however, if the object's color were known and/or readily identifiable as the target without need for motion analysis. Then it would make sense to segregate the object from its background before determining in which direction it moved, since this would eliminate the processing of irrelevant motion information.

Figure 11 illustrates the possible functional relationships between color and motion processing for the stimuli employed here. Figure 11A illustrates the relationship that, we argue, can be rejected: automatic grouping, or prefiltering, of local features by color/luminance prior to motion processing. Figures 11B, 11C, and 11D describe three relationships that our results support. In Figure $11 \mathrm{~B}$, there are equal numbers of target and distractor dots, and the motion system integrates local feature information regardless of color/luminance. In Figure 11C, on the other hand, the target elements are in a minority and, when different in color/luminance from the distractor, are segregated prior to motion processing; they pop out. In Figure 11D, the target dots do not pop out, but because their color/luminance is known, they can be selectively attended to and segregated for motion processing.

\section{CONCLUSION}

In multielement moving stimuli, elements with different colors and/or luminance polarities are not automatically grouped into separate color/luminance maps for motion processing, even if it is beneficial to do so. Only when there are static form cues to the target, or when the target color/luminance is known to the observer and selectively attended to, is motion perception facilitated when target and distractors differ in their color/luminance properties.

\section{REFERENCES}

Albright, T. D. (1992). Form-cue invariant motion processing in primate visual cortex. Science, 255, 1141-1143.

Berger, B. C., \& McLeod, P. (1996). Display density influences visual search for conjunctions of movement and orientation. Journal of Experimental Psychology: Human Perception \& Performance, 22, $114-$ 121.

Britten, K. H., Shadlen, M. N., Newsome, W. T., \& Movshon, J. A. (1992). The analysis of visual motion: A comparison of neuronal and psychophysical performance. Journal of Neuroscience, 12, 4745-4765.

Cavanagh, P., \& Favreau, O. E. (1985). Color and luminance share a common motion pathway. Vision Research, 25, 1595-1601.

Croner, L. J., \& Albright, T. D. (1994). Segmentation by colour im- proved performance on a motion discrimination task [Abstract]. Investigative Ophthalmology \& Visual Science, 35, S1643.

Croner, L. J., \& Albright, T. D. (1997). Image segmentation enhances discrimination of motion in visual distractor. Vision Research, 37, 1415-1427.

Cropper, S. J., \& Derrington, A. M. (1996). Rapid colour-specific detection of motion in human vision. Nature, 379, 72-74.

Culham, J. C., Brandt, S. A., Cavanagh, P., Kanwisher, N. G., Dale, A. M., \& Tootell, R. B. (1998). Cortical fMRI activation produced by attention tracking of moving targets. Journal of Neurophysiology, 80, 2657-2670.

Driver, J., \& MCLeOd, P. (1992). Reversing visual search asymmetries with conjunctions of movement and orientation. Journal of Experimental Psychology: Human Perception \& Performance, 18, 22-33.

EDWARds, M., \& BADCock, D. R. (1994). Global motion perception: Interaction of on and off pathways. Vision Research, 34, 2849-2858.

EDWARDS, M., \& BADCock, D. R. (1996). Global-motion perception: Interaction of chromatic and luminance targets. Vision Research, 36, 2423-2431.

Green, M. (1989). Color correspondence in apparent motion. Perception \& Psychophysics, 45, 15-20.

Livingstone, M. S., \& Hubel, D. H. (1988). Segregation of from, color, movement and depth: Anatomy, physiology and perception. Science, 240, 740-749.

MARQUARDT, D. W. (1963). An algorithm for least-squares estimation of non-linear parameters. Journal of the Society of Industrial \& Applied Mathematics, 11, 431-441.

McLeod, P., Driver, J., \& CRISP, J. (1988). Visual search for a conjunction of movement and form is parallel. Nature, 332, 154-155.

McLeod, P., Driver, J., Dienes, Z., \& Crisp, J. (1991). Filtering by movement in visual search. Journal of Experimental Psychology: Human Perception \& Performance, 17, 55-64.

Morgan, M. J., \& InGLe, G. (1994). What direction of motion do we see if luminance but not colour contrast is reversed during displacement? Psychophysical evidence for a signed-colour input to motion detection. Vision Research, 34, 2527-2535.

Mullen, K. T., \& BaKer, C. L. (1985). A motion aftereffect from an isoluminance stimulus. Vision Research, 25, 685-688.

MüLler, H. J., \& Found, A. (1996). Visual search for conjunctions of motion and form: Display density and asymmetry reversal. Journal of Experimental Psychology: Human Perception \& Performance, 22, 122-132.

Nakayama, K., \& Silverman, G. H. (1986). Serial and parallel processing of visual feature conjunctions. Nature, 320, 264-265.

Palmer, J., Mobley, L. A., \& Teller, D. Y. (1993). Motion at isoluminance: Discrimination/detection ratios and the summation of luminance and chromatic targets. Journal of the Optical Society of America A, 10, 1353-1362.

Papathomas, T. V., Gorea, A., \& Julesz, B. (1991). Two carriers for motion perception: Color and luminance. Vision Research, 31, 1883 1891.

Press, W. H., Teukolsky, S. A., Vetterling, W. T., \& Flannery, B. P. (1992). Numerical recipes in C (2nd ed.). New York: Cambridge University Press.

Pylyshyn, Z W., \& Storm, R. W. (1988). Tracking of multiple independent targets: Evidence for a parallel tracking mechanism. Spatial Vision, 3, 1-19.

Ramachandran, V. S., \& Gregory, R. L. (1978). Does color provide an input to motion perception? Nature, 275, 55-56.

Saito, H., Tanaka, K., Isono, H., Yasuda, M., \& Mikami, A. (1989). Directionally selective response of cells in the middle temporal area (MT) of the macaque monkey to the movement of equiluminous opponent color stimuli. Experimental Brain Research, 75, 1-14.

Schiller, P. H., Logothetis, N. K., \& Charles, E. R. (1990). Functions of the colour-opponent and broad-band channels of the visual system. Nature, 343, 68-70.

SNOWDEN, R. J., \& EDMUnds, R. (1999). Color and polarity contributions to global motion perception. Vision Research, 39, 1813-1822. 
Stoner, G. R., \& Albright, T. D. (1993). Image segmentation cues in motion processing: Implications for modularity in vision. Journal of Cognitive Neuroscience, 5, 129-149.

WeIBULL, W. (1951). A statistical distribution function of wide applicability. Journal of Applied Mechanics, 18, 293-297.

Yoshizawa, T., Mullen, K. T., \& BaKer, C. L., JR. (2000). Absence of a chromatic linear motion mechanism in human vision. Vision Research, 40, 1993-2010.

ZEKI, S. M., \& SHIPP, S. (1988). The functional logic of cortical connections. Nature, $\mathbf{3 3 5}, 311-317$.

\section{NOTE}

1. The Weibull function is $p(c)=1-(1-\gamma) \exp \left[-(c / \alpha)^{\beta}\right]$, where $c$ is target strength, $\gamma$ is guessing rate, $\alpha$ is threshold, and $\beta$ is slope of the psychometric function (Weibull, 1951). In our two-alternative forcedchoice task, the guessing rate was .5. The proportion correct at threshold was determined when $c=\alpha$, which is .82 .

(Manuscript received June 25, 1998;

revision accepted for publication July 31,2000 .) 